Canadian Journal of Applied Linguistics

\title{
Cours de français langue seconde pour personnes immigrantes à Québec : portrait des habiletés orales en fin de parcours
}

\author{
Suzie Beaulieu, Leif Michael French, Javier Bejarano et Kristin Reinke
}

Volume 24, numéro 3, automne 2021

URI : https://id.erudit.org/iderudit/1084808ar

DOI : https://doi.org/10.37213/cjal.2021.31120

\section{Aller au sommaire du numéro}

\section{Éditeur(s)}

University of New Brunswick

\section{ISSN}

1920-1818 (numérique)

Découvrir la revue

\section{Citer cet article}

Beaulieu, S., French, L., Bejarano, J. \& Reinke, K. (2021). Cours de français langue seconde pour personnes immigrantes à Québec : portrait des habiletés orales en fin de parcours. Canadian Journal of Applied Linguistics / Revue canadienne de linguistique appliquée, 24(3), 1-29.

https://doi.org/10.37213/cjal.2021.31120

\section{Résumé de l'article}

Les programmes de formation linguistique à l'intention de personnes immigrantes adultes font partie intégrante des initiatives politiques et éducatives mises de l'avant par plusieurs pays pour favoriser leur intégration à la société d'accueil. Cependant, peu d'attention empirique leur a été accordée pour observer comment s'y opère le développement langagier. Pour combler cette lacune, cette étude exploratoire et descriptive documente, pour la première fois, les habiletés orales de personnes immigrantes terminant une formation linguistique financée par un gouvernement local. Nous avons examiné la compétence globale à l'oral, l'aisance perçue et la compréhensibilité en français d'immigrants adultes $(\mathrm{N}=31)$ dans la ville de Québec inscrits au dernier niveau du Programme d'intégration linguistique pour les immigrants (PILI). Le niveau de compétence orale globale a été obtenu en utilisant une tâche d'imitation orale (EIT) (Tracy-Ventura et coll., 2014). Pour mesurer l'aisance perçue et la compréhensibilité des participants, nous avons conçu deux tâches monologiques qui imposaient différentes contraintes linguistiques et cognitives au niveau du traitement de l'information. Les échantillons de paroles ont été évalués par 13 juges experts francophones, en utilisant une échelle de Likert à 9 points. Les résultats montrent que les habiletés orales des finissants du PILI sont très hétérogènes, et que ce sont les personnes qui avaient étudié le français pendant plusieurs années avant d'immigrer qui ont généralement de meilleurs résultats sur les trois construits.
Copyright @ Suzie Beaulieu, Leif Michael French, Javier Bejarano, Kristin Reinke, 2021
Ce document est protégé par la loi sur le droit d'auteur. L’utilisation des services d'Érudit (y compris la reproduction) est assujettie à sa politique d'utilisation que vous pouvez consulter en ligne.

https://apropos.erudit.org/fr/usagers/politique-dutilisation/ 


\title{
Cours de français langue seconde pour personnes immigrantes : portrait des habiletés orales en fin de parcours
}

\author{
Suzie Beaulieu \\ Université Laval \\ Leif Michael French \\ Sam Houston State University \\ Javier Bejarano \\ Université Laval \\ Kristin Reinke \\ Université Laval
}

\begin{abstract}
Résumé
Les programmes de formation linguistique à l'intention de personnes immigrantes adultes font partie intégrante des initiatives politiques et éducatives mises de l'avant par plusieurs pays pour favoriser leur intégration à la société d'accueil. Cependant, peu d'attention empirique leur a été accordée pour observer comment s'y opère le développement langagier. Pour combler cette lacune, cette étude exploratoire et descriptive documente, pour la première fois, les habiletés orales de personnes immigrantes terminant une formation linguistique financée par un gouvernement local. Nous avons examiné la compétence globale à l'oral, l'aisance perçue et la compréhensibilité en français d'immigrants adultes $(\mathrm{N}=31)$ dans la ville de Québec inscrits au dernier niveau du Programme d'intégration linguistique pour les immigrants (PILI). Le niveau de compétence orale globale a été obtenu en utilisant une tâche d'imitation orale (EIT) (Tracy-Ventura et coll., 2014). Pour mesurer l'aisance perçue et la compréhensibilité des participants, nous avons conçu deux tâches monologiques qui imposaient différentes contraintes linguistiques et cognitives au niveau du traitement de l'information. Les échantillons de paroles ont été évalués par 13 juges experts francophones, en utilisant une échelle de Likert à 9 points. Les résultats montrent que les habiletés orales des finissants du PILI sont très hétérogènes, et que ce sont les personnes qui avaient étudié le français pendant plusieurs années avant d'immigrer qui ont généralement de meilleurs résultats sur les trois construits.
\end{abstract}

\begin{abstract}
Language training programs for adult immigrants are an integral part of the political and educational landscape for many immigrant-receiving countries. However, little empirical research has addressed how language skills develop within these programs. To fill this gap, this exploratory and descriptive study documents, the oral skills of immigrants completing a language training program funded by a local government. We examined the global oral proficiency, perceived fluency and comprehensibility of adult immigrants $(\mathrm{N}=31)$ enrolled in the highest level of Québec's French language training program: le Programme
\end{abstract}


d'intégration linguistique pour les immigrants (PILI). Global proficiency was measured using an oral imitation task (EIT, Tracy-Ventura et al., 2014). Two monological tasks that imposed different linguistic and cognitive constraints were used to assess participants' fluency and comprehensibility. The speech samples were rated by 13 expert French-speaking judges, using a 9-point Likert scale. The results showed that the oral skills of PILI graduates are heterogeneous, and that participants who had studied French for several years before immigrating demonstrated better results across all three constructs.

\section{Cours de français langue seconde pour personnes immigrantes : Portrait des habiletés orales en fin de parcours}

\section{Introduction}

Le Canada s'est développé au fil de vagues successives d'immigration qui ont permis et permettent encore de combler d'importants besoins économiques et sociaux (Gauthier, 2016). Il en est de même pour la province de Québec où la présence de communautés ethnolinguistiques s'est accrue de manière importante au cours des quinze dernières années (Institut de la statistique du Québec, 2019). En effet, le Québec mise sur un accroissement des communautés immigrantes pour faire face au vieillissement de la population, pour combler une rareté de main-d'œuvre et pour contribuer à la vitalité du français comme langue commune (MIFI, 2015a). Ces diverses contributions citoyennes, économiques et culturelles à la société québécoise dépendent cependant de l'intégration réussie des nouveaux arrivants, qui repose largement sur la maîtrise de la langue française (MIFI, 2015b).

Les nouveaux arrivants qui ne maitrisent pas suffisamment le français à leur arrivée peuvent bénéficier gratuitement de cours de français langue seconde $(\mathrm{Lx})^{1}$ grâce au Programme d'intégration linguistique pour les immigrants (PILI), dont la mission s'inscrit dans une logique de pleine participation à la société québécoise et « vise à soutenir la personne immigrante dans sa démarche d'apprentissage du français, langue d'intégration et de cohésion sociale, afin qu'elle puisse s'intégrer à la société québécoise francophone » (Amireault et Lussier, 2008, p. 3). L'une des finalités de cette formation culturelle et linguistique est l'atteinte du niveau 8 à l'oral de l'Échelle québécoise des niveaux de compétence en français des personnes immigrantes adultes (voir Gouvernement du Québec, 2011), niveau considéré comme le seuil d'autonomie langagière à atteindre pour pouvoir occuper un emploi ou poursuivre des études en français (MIFI, 2019).

Étant donné l'importance de la maîtrise du français pour l'intégration socioprofessionnelle dans la province de Québec et plus particulièrement dans la ville de Québec, où, selon le dernier recensement, près de $97 \%$ des répondants rapportent que le français est la première langue officielle qu'ils utilisent au quotidien (Statistique Canada, 2017), il est essentiel de dresser un portrait des habiletés à l'oral des nouveaux arrivants appartenant à la catégorie immigrant économique qui ont complété le PILI. En effet, bien que ceux-ci se soient vus accordés l'entrée au Québec en vertu de leurs qualifications professionnelles, la démonstration de leur habileté à s'exprimer à l'oral avec efficacité est déterminante pour l'accès à l'emploi (Plante, 2011). 


\section{État de la question}

\section{Cadres et référentiels de compétences langagières}

Plusieurs pays accueillant un nombre important de personnes immigrantes proposent des cours de langues afin de leur permettre de développer leurs compétences langagières dans la langue majoritaire de la société, ce qui favorisera leur intégration socioprofessionnelle. Plusieurs de ces nations se sont dotées de référentiels pour baliser le parcours d'apprentissage de la ou des langue.s officielle.s de la communauté d'accueil, par exemple le Cadre européen commun de référence pour les langues (CECR) qui existe pour plusieurs langues parlées en Europe, ou encore l'American Council on the Teaching of Foreign Languages (ACTFL) pour l'anglais aux États-Unis. Au Canada, ce sont les Canadian Language Benchmarks (CLB) qui font office de référence. À leur parution en 1996, seule une progression des apprentissages pour l'anglais langue seconde existait (Immigration, Réfugiés et Citoyenneté Canada, 2012). Inspirés de ce cadre, deux référentiels différents pour le français Lx sont apparus au tournant des années 2000. En effet, une traduction des CLB a été effectuée qui a donné lieu aux Standards linguistiques canadiens (depuis 2012, les Niveaux de compétence linguistique canadiens), publiés en 2002 (Immigration, Réfugiés et Citoyenneté Canada, 2012). À notre connaissance, la version traduite en français des CLB n'a jamais réellement été reconnue ou utilisée puisqu'une adaptation des CLB pour le Québec avait été effectuée et publiée en 2000 et s'était déjà imposée comme référence dans la province : Les Niveaux de compétence en français langue seconde pour les immigrants adultes (Gouvernement du Québec, 2011). Depuis 2011, on balise la progression des apprentissages en fonction de L'Échelle québécoise des niveaux de compétence en français des personnes immigrantes adultes, une mise à jour du référentiel paru en 2000.

Depuis l'adoption de cette échelle, « le MIDI [maintenant MIFI], le MEES [ministère de l'Éducation et de l'Enseignement supérieur] et leurs partenaires considèrent que le niveau 8 (sur un total de 12) de l'Échelle québécoise des niveaux de compétence en français des personnes immigrantes adultes correspond au seuil qui permet aux nouveaux arrivants de poursuivre des études en français et d'accéder à la plupart des emplois » (Conseil supérieur de la langue française, 2018, p.V). Or, il est intéressant de noter qu'aucune explication n'est offerte quant aux fondements empiriques (p.ex. exploitation de corpus de langue au travail) sur lesquels repose cette décision.

En 2017, la vérificatrice générale du Québec a mené un audit de performance du PILI. De cette évaluation, les médias retiennent que « la francisation des immigrants est un échec » (Richer, 2017), puisque la vérificatrice générale relève notamment que « [p]armi les personnes ayant participé aux programmes de formation du ministère, la vaste majorité n'a pas atteint le seuil d'autonomie langagière » (Vérificateur général du Québec, 2017, p. 14). En effet, entre 2011 et 2015 , le taux de réussite de ce niveau à l'oral se situe entre 9,1 et $16,3 \%$. Par ailleurs, la vérificatrice reproche que « [m]algré le faible taux d'atteinte du seuil d'autonomie langagière, tant pour le programme régulier que pour le programme adapté, aucune évaluation de programme n'a été réalisée » (p. 23). À cette lacune, s'ajoute aussi la même observation que la nôtre, soit que l'identification du niveau 8 comme représentant le stade d'autonomie langagière ne semble pas avoir reposé sur une analyse des besoins menée en milieux de travail ou universitaires, mais plutôt sur un processus d'adéquation avec d'autres échelles reconnues (p.ex. niveau B2 du Cadre européen 
commun de référence considéré comme le niveau où l'apprenant est un utilisateur indépendant), qui n'est pas rendu public.

\section{Évaluation empirique des programmes de formation linguistique pour immigrants adultes}

Bien que les programmes de formation linguistique à l'intention d'immigrants adultes fassent partie des initiatives politiques et éducatives mises de l'avant par plusieurs pays, et ce, depuis de nombreuses décennies, peu d'attention empirique leur a été accordée pour observer comment s'y opère le développement langagier (Adami, 2012; Burns et Roberts, 2010). À notre connaissance, seul le ministère de la Citoyenneté et de l'Immigration Canadien a documenté le développement langagier effectué par les personnes immigrantes recevant des cours d'anglais (Language instruction for Newcomers to Canada, $L I N C$ ) financés par le gouvernement fédéral.

Le programme LINC offert dans 7 provinces canadiennes a été évalué suivant un devis pré-post test. Les chercheurs ont comparé le niveau de compétence, selon les CBL, dans les quatre habiletés chez des personnes qui ont suivi la formation LINC $^{2}$ et un groupe témoin constitué de personnes qui s'étaient inscrites à LINC (et qui ont fait le pré-test), mais qui n'ont pas suivi la formation. Les résultats révèlent que les participants qui ont suivi le programme LINC, et notamment ceux qui ont assisté à plus de 1000 heures de cours LINC, ont progressé davantage que les personnes qui ne l'ont pas suivi, mais seule la différence entre la lecture et l'écriture était significativement différente. Les chercheurs ont identifié un facteur, autre que la participation au programme LINC, pouvant expliquer la présence de scores similaires à l'oral : la durée du temps de résidence. Plus leur temps de résidence au Canada était long, plus leurs habiletés en compréhension et production orales étaient semblablement élevées (IRCC, 2011) Bien que le IRCC offre également des Cours de langue pour les immigrants au Canada (CLIC), ce programme ne semble pas avoir été évalué de la même manière que le LINC, seule des données concernant le profil des personnes s'étant inscrites et ayant terminé le programme ayant été recueillies (IRCC, 2010).

À ce type d'évaluation, s'ajoutent quelques enquêtes menées auprès d'immigrants adultes pour sonder leurs perceptions quant aux programmes de formation linguistique offerts par les autorités locales. De manière générale, la majorité a rapporté être satisfaite de l'enseignement reçu, avoir fait des gains langagiers significatifs et avoir acquis des connaissances utiles sur la société d'accueil. Néanmoins, beaucoup estiment qu'ils n'ont pas développé les compétences linguistiques nécessaires pour entreprendre des études postsecondaires ou entrer sur le marché du travail. Ces constats ont été observés dans plusieurs milieux, entre autres au Québec chez les participants ayant terminé le PILI à Saguenay (Carrier-Giasson, 2017) et à Montréal (Amireault et Lussier, 2008). Il ressort également de l'ensemble de ces études que les participants perçoivent leurs habiletés à l'écrit comme étant meilleures que celles à l'oral; pour ce qui est de l'oral, ils perçoivent leurs compétences en compréhension meilleure que celle en production. Ces résultats correspondent aux tendances relevées par le IRCC (2011) et corroborent les observations issues de l'étude ethnographique de Roussel (2018) menée auprès de six nouveaux arrivants établis dans une petite localité majoritairement francophone de l'Est-du-Québec. Bien que tous les participants de cette étude s'étaient vus reconnaître la compétence linguistique nécessaire pour être admis dans un programme universitaire offert en français, 
ils éprouvaient des difficultés lors d'interactions orales quotidiennes avec des natifs qui disaient ne pas les comprendre ou préférer leur parler en anglais.

À notre connaissance, aucune étude n'a encore documenté, à partir de données effectives (p.ex. tests ou échantillons de parole), la compétence linguistique de finissants d'une formation linguistique offerte aux personnes immigrantes, formation dont le but est de favoriser l'intégration socioprofessionnelle à la société québécoise. Nous nous concentrons sur l'évaluation de leurs habiletés orales, celles-ci étant fortement corrélées à des indicateurs d'intégration à la société d'accueil, tels que l'accès aux emplois correspondant à la formation professionnelle obtenue avant l'immigration (Plante, 2011), l'utilisation optimale de l'ensemble des services sociaux offerts dans la société d'accueil, dont les services de santé (Lebrun, 2012) ou encore l'existence de réseaux sociaux diversifiés (Laliberté, 2019).

\section{Les habiletés orales}

La question des habiletés orales est cruciale pour l'accès à l'emploi. Plusieurs employeurs les considèrent comme un critère de sélection et l'abordent soit sous l'angle de

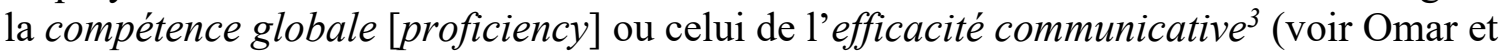
coll., 2012). En effet, l'obtention d'un emploi et même l'admission à un programme universitaire requiert régulièrement la réussite d'un test de compétence globale (Dustmann et Van Soest, 2002; Ortiz et coll., 2016). Bien que plusieurs définitions de la compétence globale existent (Leclerqc et Edmonds, 2014), nous retenons celle de Shin (2013) qui ressemble la plus à l'opérationnalisation que font les employeurs et qui veut que la compétence globale corresponde aux connaissances que le locuteur a du code de la langue cible (p.ex. connaissance de mots, de structures syntaxiques variées). Elle se mesure typiquement à l'aide d'un test standardisé (De Jong et coll., 2012).

D'autres employeurs considèrent également les habiletés orales pour la sélection des candidates, mais en utilisant plutôt un critère plus subjectif, soit : « communiquer de façon efficace » (Harrisson, 2014). Typiquement, les employeurs se fient à leur perception du niveau d'aisance et de compréhensibilité des candidats dans les entrevues d'embauche pour sélectionner la personne retenue (Harrison, 2014; Ramjattan, 2019) ${ }^{4}$. Ces deux construits sont bien définis dans la littérature en linguistique appliquée. L'aisance perçue est une mesure subjective de l'aisance énonciative, c'est-à-dire une impression que les processus cognitifs sous-tendant la planification de la parole et sa production fonctionnent sans peine (Segalowitz, 2010). L'aisance d'un locuteur peut être influencée par plusieurs facteurs, dont les exigences de la situation de communication (Javad Ahmadian et coll., 2015). Par exemple, si le temps de préparation demeure le même, les locuteurs sont perçus comme ayant plus d'aisance à l'oral dans les tâches de lecture à voix haute que dans les tâches requérant une production spontanée, la première imposant moins de contraintes cognitives que la seconde (Bradlow et coll., 2017).

La compréhensibilité est elle aussi un jugement subjectif qui fait référence au degré d'effort cognitif que l'auditeur perçoit devoir fournir pour comprendre la personne (Derwing et Munro, 2009). Tout comme pour l'aisance perçue, on observe que la nature de la production semble influencer le jugement de compréhensibilité des auditeurs. En effet, moins la tâche est cognitivement complexe (p.ex. raconter une anecdote personnelle), plus le jugement de compréhensibilité est clément et, en revanche, plus la tâche impose des 
contraintes linguistiques (p.ex. exigence d'utiliser certains mots) ou cognitives (p.ex. plusieurs éléments à planifier), plus le jugement est sévère (Crowther, 2020).

L'aisance perçue et la compréhensibilité sont deux construits distincts, mais interreliés : ainsi, la grande présence de pauses et d'hésitations entrainerait un effort cognitif important de la part de l'interlocuteur, affectant la compréhensibilité (Suzuki et Kormos, 2019). Dans le cas de ces deux construits, la nature de la tâche de production influence le jugement de la performance à l'oral. Par contre, la compétence globale dans la langue ne serait pas corrélée à la compréhensibilité ni à l'aisance (Sato et McNamara, 2019; Suzuki et Kormos, 2019). Il est donc essentiel de les étudier de façon séparée pour dresser un portrait détaillé et nuancé de la compétence linguistique à l'oral.

\section{La présente étude}

Le PILI est le programme de formation linguistique offert par le gouvernement québécois aux nouveaux arrivants qui désirent améliorer leurs compétences en français, seule langue officielle de la province de Québec. À l'automne 2017, ce programme a été sévèrement critiqué par la vérificatrice générale, notamment parce que peu de personnes immigrantes finissaient la formation et, par conséquent, c'est donc un nombre très peu élevé qui obtient le niveau d'autonomie langagière jugé nécessaire pour poursuivre des études ou travailler en français. De plus, des personnes ayant suivi le PILI sont d'avis qu'au terme de leur formation, leur compétence à l'oral n'était pas aussi élevée que celle à l'écrit, voire même qu'elle était insuffisante pour répondre à leurs besoins socioprofessionnels. Cependant, aucune étude, à notre connaissance, n'a encore tenté de documenter à partir de données effectives les habiletés orales de ce groupe singulier de personnes terminant le PILI. C'est là l'objectif de notre étude. Plus spécifiquement, nous cherchons à répondre aux questions de recherche suivantes :

1. Quelle est la compétence globale des personnes immigrantes finissant le PILI ?

2. Quel est le niveau d'aisance perçue de personnes immigrantes finissant le PILI dans deux conditions (lecture à voix haute et production spontanée) ?

3. Quel est le niveau de compréhensibilité des personnes immigrantes finissant le PILI dans deux conditions (lecture à voix haute et production spontanée)?

\section{Méthodologie}

\section{Le cours du PILI menant à l'obtention du niveau 8}

Cette étude s'est intéressée de façon précise aux apprenants qui, contrairement à la tendance générale observée par la vérificatrice générale du Québec (2017), ont poursuivi leurs parcours au PILI jusqu'au niveau terminal. Ce niveau correspond au FIA-330-4, cours qui mène au développement des compétences du niveau 8 à l'oral de L'échelle québécoise des niveaux de compétence en français. Les cours FIA regroupe un maximum de 20 apprenants, tous scolarisés (11 années de scolarisation ou plus). L'horaire hebdomadaire est constitué de 20 heures d'enseignement formel en classe assurées par un enseignant et de 10 heures de soutien linguistique et culturel offertes par un animateur, habituellement un étudiant de premier cycle inscrit au baccalauréat en enseignement du français langue seconde ou une discipline connexe L'enseignement préconisé est basé sur 
l'approche communicative, c'est-à-dire on attend de l'apprenant qu'il participe activement puisque la langue est d'abord et avant tout perçue comme un outil de communication (Gouvernement du Québec, 2006). Le progrès des apprentissages est évalué tout au long de la formation (évaluation formative et sommative à la fin de chaque niveau) et un relevé des apprentissages détaillant si les objectifs du niveau ont été atteints est produit à la dernière semaine du cours. Il est important de noter que même si un apprenant n'a pas atteint l'ensemble des objectifs du niveau, il peut passer au niveau suivant s'il le souhaite.

L'objectif général du niveau 8, en production orale, est le suivant : « Communique[r] de façon autonome, quand la situation est prévisible ou partiellement prévisible, dans des conversations ou des présentations informelles sur des thèmes concrets ou certains sujets d'intérêt général liés à des besoins courants ou particuliers à des groupes de personnes ». (Gouvernement du Québec, 2011, p.26). Les 17 indicateurs de performance de ce niveau incluent principalement des connaissances du code linguistique (Annexe A). Dans la majorité des cas, des attentes en précision [accuracy] sont clairement énoncées (p.ex. raconte un événement en utilisant adéquatement le passé composé, l'imparfait et généralement le plus-que-parfait; emploie correctement des verbes appropriés pour exprimer des sentiments et des opinions - la mise en relief en gras est présente dans l'original). On parle minimalement et de façon implicite de compréhensibilité dans un seul descripteur (emploie des structures de phrase variées comportant plusieurs erreurs qui toutefois n'entravent que rarement la communication). Un descripteur précise également que des lacunes en aisance perçue peuvent être tolérées, si elles sont peu fréquentes (s'exprime en s'autocorrigeant à l'occasion).

\section{Les participants}

Après avoir obtenu l'approbation du comité déontologique de notre université, le ministère de l'Immigration, de la Francisation et de l'Intégration nous a également permis de recruter des participants pendant les heures consacrées au soutien linguistique et culturel des cours FIA-330-4 et de collecter des échantillons de parole des personnes se portant volontaire pour participer à notre étude. Il est a noté que le ministère nous a accordé la permission de nous entretenir avec la responsable des cours pour en savoir davantage sur le programme, mais il ne nous était pas permis d'observer les cours ni de consulter le matériel utilisé en classe.

Au total, 31 participants ont accepté de prendre part à cette étude $(18 \mathrm{~F}, 13 \mathrm{H})$. Comme aucune différence significative n'a été observée en ce qui concerne les caractéristiques individuelles ou en ce qui concerne la performance sur les trois construits, nous les considérons comme un seul groupe de participants. Le Tableau 1 présente le profil de ces participants. Tous étaient résidants de la ville de Québec depuis, en moyenne, un peu moins d'un an (étendue : de 3 mois à 3 ans). Ils avaient entre 22 à 54 ans (âge moyen : 34,5 ans). Les langues maternelles et dominantes parlées par les participants étaient l'espagnol $(n=12)$, l'arabe $(n=5)$, le portugais $(n=4)$, le dari $(n=2)$ et une seule personne locutrice pour chacune des langues suivantes : l'anglais, le kinyarwanda, le kirundi, le malinké, le pamir, le roumain, le sango et le vietnamien. Les participants avaient différents niveaux d'éducation (universitaire $1^{\mathrm{e}}$ cycle, $\mathrm{n}=22$; collégial, $\mathrm{n}=6$, et secondaire, $\mathrm{n}=3$ ). Leur expérience d'apprentissage du français avant leur arrivée à Québec variait beaucoup (voir grand écart type dans le Tableau 1) : alors que certains n'avaient jamais étudié cette 
langue avant d'immigrer, d'autres l'avaient déjà étudié; un groupe restreint de participants avait même été scolarisé en français. L'ensemble des participants a déclaré utiliser le français au quotidien dans des échanges à l'oral ou à l'écrit hors de la salle de classe en moyenne 2,5 heures par jour et être exposés au français hors des cours du programme (c.à.d., exposition passive par l'intermédiaire, par exemple, de films ou de séries télévisées), également en moyenne 2,5 heures par jour. Les grands écarts types indiquent que les participants variaient grandement en termes d'usage et d'exposition au français au quotidien.

\section{Tableau 1}

Caractéristiques des participants

\begin{tabular}{lc}
\hline Sexe & Homme - 13; Femme - 18 \\
\hline Moyenne âge (écart type) & $34,4(8,1)$ \\
Moyenne résidence au Québec - en mois & $11,5(7,8)$ \\
Moyenne études en français - en années & $3,1(4)$ \\
Moyenne exposition quotidienne - en heures & $2,5(1,45)$ \\
Moyenne usage quotidien - en heures & $2,5(2,5)$ \\
\hline
\end{tabular}

\section{Les construits mesurés}

Pour dresser un portrait nuancé des habiletés orales des personnes complétant le niveau terminal du PILI, nous avons recueilli des données nous informant sur leur compétence globale, leur aisance perçue et leur compréhensibilité.

\section{La compétence globale}

Pour mesure la compétence globale de nos participants, la version française de l'Elicited Imitation Test (EIT), disponible sur IRIS Digital Repository (http://www.irisdatabase.org), a été retenue (Tracy-Ventura et coll., 2014). L'EIT est composé de 30 énoncés, dont la longueur et la complexité linguistique augmentent au fil du test (de 7 à 19 syllabes, phrases simples à phrases coordonnées à phrases subordonnées). Les participants doivent répéter ces énoncés le plus fidèlement possible. À noter que pour éviter la répétition par cœur, une pause de 2 secondes est insérée entre la fin de chaque énoncé et le son, un bip sonore de 0,5 seconde, incitant les participants à commencer chaque répétition. L'EIT a été conçu et validé en 5 autres langues : anglais, allemand, japonais (Ortega et coll., 2002), mandarin (Wu et Ortega, 2013) et espagnol (Bowden, 2016). Les résultats d'une récente méta-analyse de Yan et coll. (2016) montrent que l'EIT a une forte validité de construit et conséquemment qu'il est une mesure fiable de compétence globale de la langue. 


\section{L'aisance perçue et la compréhensibilité}

Pour mesurer l'aisance perçue et la compréhensibilité des participants, nous avons conçu deux tâches monologiques qui imposaient différentes contraintes linguistiques et cognitives au niveau du traitement de l'information. La première est une tâche de lecture à voix haute, inspirée de Lindemann (2003). Le texte à lire de manière naturelle était le message d'accueil d'une boîte vocale d'un restaurant et contenait une centaine de mots simples (Annexe B). Cette tâche était peu linguistiquement et cognitivement complexe puisqu'aucune conceptualisation ni formulation des énoncés (Levelt, 1989) n'était requise de la part des participants. La deuxième tâche était une production libre, mais guidée, adaptée de Dupere (2018). Les participants devaient prétendre qu'ils devaient répondre à la question d'un client (joué par un assistant de recherche) en se servant des indications laissées sous forme de mots clés par un collègue (Annexe C). Cette tâche imposait davantage de contraintes linguistiques et cognitives (c.à.d., que les participants n'étaient pas entièrement libres de répondre avec leurs propres idées); ils devaient partiellement conceptualiser et formuler le message.

\section{Collecte de données}

La collecte des échantillons de parole s'est déroulée avec un membre de l'équipe de recherche dans un local insonorisé près de la salle de classe des participants, à un moment qui leur convenait. En premier lieu, les participants ont effectué la tâche de lecture à voix haute. Chaque participant disposait d'une période de préparation de 2 minutes pour prendre connaissance du message. Ils ont aussi reçu la consigne de lire à voix haute de manière à ce que le message vocal soit perçu comme étant naturel. Par la suite, on a demandé aux participants de prendre part à la tâche de production libre, mais guidée. Encore une fois, deux minutes ont été accordées pour permettre au participant de se préparer. Ensuite, les participants ont eu une période de familiarisation avec le EIT (contenant 5 phrases à répéter) et ont tenté de reproduire les 30 items du EIT. Pour finir, les participants ont complété un questionnaire sociodémographique. La collecte a duré entre 25 et 45 minutes, les participants pouvant prendre des pauses entre chacune des tâches à leur gré.

\section{Analyse des données orales recueillies}

À l'instar des études qui ont fait usage du EIT, la performance des participants a été enregistrée et a ensuite été évaluée à l'aide d'une grille à 5 points (Figure 1), par trois juges experts. Les juges avaient préalablement suivi une formation avec la première auteure pour se familiariser avec la grille d'évaluation (aussi trouvée sur http://www.iris-database.org) et uniformiser leur jugement. 


\title{
Figure 1
}

Critères d'évaluation utilisés pour évaluer la performance au EIT (notre traduction de Ortega et coll., 2002).

\author{
4 = répétition parfaite \\ 3 = Répétition précise du contenu avec quelques changements de forme \\ (non grammaticaux ou grammaticaux) \\ $2=$ Changements de contenu ou de forme qui affectent le sens \\ $1=$ Répétition de la moitié du stimulus ou moins \\ $0=$ silence, un seul mot répété ou répétition inintelligible
}

Ainsi, deux juges ont attribué un score entre 0 et 4 à chacun des trente enregistrements produits par chaque participant, pour un score total maximum de 120 . L'accord interjuges a été établi à 0,88 . Les cas de désaccord ont été résolus par le troisième juge. Le score sur 120 des participants a été utilisé pour discuter de la compétence globale des participants.

Les enregistrements issus de la tâche de lecture et de la tâche de production spontanée ont été traités pour réduire la présence de bruits de fond, pour enlever les interventions d'un membre de l'équipe de recherche ou pour enlever les premières secondes contenant des hésitations pour amorcer la tâche. Les participants ont produit des échantillons de parole de longueur variant de 19,6 à 54,3 secondes ${ }^{5}$. Pour éviter d'introduire des biais dans l'évaluation en raison de la durée des extraits, tous les enregistrements ont été raccourcis à une vingtaine de secondes (en nous assurant qu'aucun mot n'était coupé). Une fois normalisés, ces échantillons de parole ont été présentés aux juges pour qu'ils évaluent la compréhensibilité et l'aisance perçue des participants. Six distracteurs représentant des voix de locuteurs de niveau inférieur au niveau 8 et supérieur à celui-ci ont été ajoutés pour s'assurer que l'étendue de l'échelle soit utilisée.

Treize locuteurs natifs du français (4 hommes, 9 femmes; étendue de l'âge : 24-35, $M=28,5$ ) inscrits dans un programme de cycle supérieur en linguistique ou en didactique des langues ont été recrutés pour évaluer les enregistrements. Ils avaient tous suivi le séminaire Enseignement des langues secondes dans lesquels les construits à l'étude ont été largement étudiés. Tous avaient plusieurs années d'expérience en enseignement du français L2, mais aucun n'avait travaillé pour le PILI auparavant. Tous ont rapporté être souvent exposés à différents accents étrangers en français. À l'instar d'études antérieures s'intéressant à la performance à l'oral en Lx (Derwing et coll., 2004 ; French, et coll., 2020 ; Isaacs et Trofimovich, 2012), les juges ont évalué à l'aide d'échelles Likert à 9 points l'aisance perçue $(1=$ très fluide, $9=$ très peu fluide $)$ et la compréhensibilité $(1=$ très facile à comprendre, $9=$ très difficile à comprendre) des 31 participants dans les deux conditions.

Avant de commencer l'évaluation, les construits à évaluer ont été présentés et expliqués aux juges par deux membres de l'équipe de recherche afin de nous assurer qu'ils comprenaient bien ce qui était attendu d'eux et d'éviter l'évaluation d'autres aspects, comme la précision à l'oral. Ils ont écouté et évalué 5 exemples avant d'évaluer l'ensemble des enregistrements. Chaque enregistrement a été écouté seulement une fois et la session a été d'une durée moyenne de 60 minutes. 


\section{Présentation des résultats}

Cette étude descriptive exploratoire avait pour objectif d'offrir un portrait détaillé des habiletés orales de personnes immigrantes terminant le PILI dans la ville de Québec.

\section{La compétence globale}

Pour répondre à la première question de recherche qui visait à décrire la compétence globale des participants qui terminent le PILI, la version française du EIT a été utilisée. Le Tableau 2 indique que les participants ont reçu un score moyen de 67,19 sur 120. L'écart type très élevé et l'étendue des résultats révèlent par ailleurs que le groupe était extrêmement hétérogène : certains participants étaient bien en deçà de la moyenne alors que certains se rapprochaient d'un score parfait.

\section{Tableau 2}

Scores au test d'imitation (EIT) des 31 participants

\begin{tabular}{lll}
\hline & $\mathrm{M}(E T)$ & Min.-Max. \\
\hline Score EIT & $67,19(22,41)$ & $20-107$ \\
\hline
\end{tabular}

Étant donné la très grande hétérogénéité du groupe, il nous était difficile de commenter de façon fiable et rigoureuse la performance du groupe. Pour pallier ce problème, nous avons utilisé une méthode statistique (median split) nous permettant de créer des groupes à la fois plus homogènes et distincts (Field, 2015). Ainsi, nous avons séparé le groupe en trois, à partir de la médiane obtenue au EIT (67) pour obtenir un portrait plus détaillé de la performance de nos participants : groupe faible (20 à 61), groupe moyen $(61,01$ à 75,23) et groupe élevé $(75,24$ à 107). La Figure 2 indique que le premier sous-groupe de participants $(n=13)$ a eu une moyenne de 48,85 , le deuxième sous-groupe $(n=8)$ de 68,63 et le dernier $(n=10)$ de 92,9 . On constate donc que même à l'intérieur des trois sous-groupes, les résultats sont très étendus, et ce encore plus chez le sous-groupe le plus faible.

\section{Figure 2}

Scores au EIT chez les sous-groupes faible, moyen et élevé

\section{Score au EIT / 120}

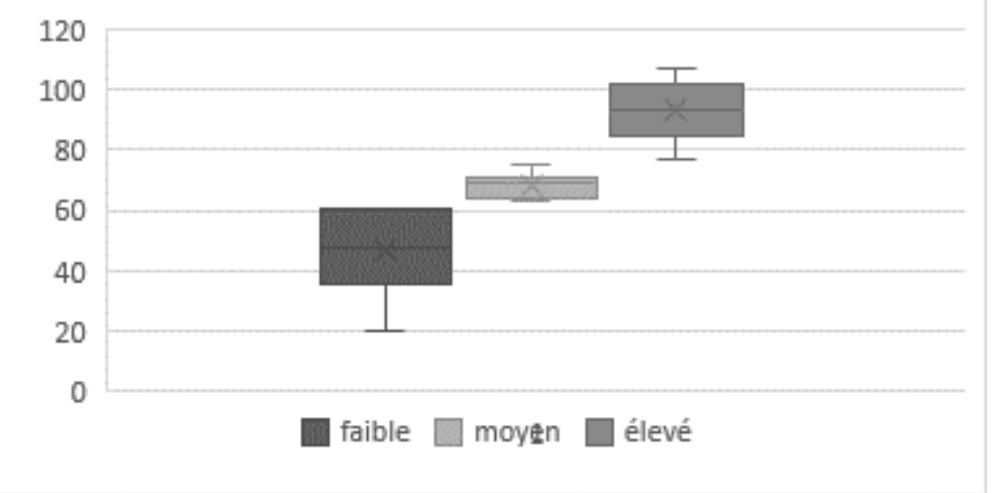


Nous avons exploré si les trois sous-groupes partageaient les mêmes caractéristiques personnelles. On peut constater au Tableau 3 que les participants des trois sous-groupes ont un âge moyen dans la trentaine et qu'ils ont rapporté un usage (utilisation productive) et un contact (exposition passive) quotidien relativement semblable. Cependant, les participants du sous-groupe le plus fort se démarquent quant à leur expérience antérieure d'apprentissage du français. Ils ont étudié, en moyenne, pendant un peu plus de 5 ans que les participants les plus faibles et pendant un peu plus de 3 ans que les participants qui ont un obtenu un résultat moyen. On remarque également que les personnes appartenant au sous-groupe le plus fort ont une durée de résidence au Québec plus courte que ceux des deux autres sous-groupes.

\section{Tableau 3}

Caractéristiques des participants dans les sous-groupes faibles, moyens et forts sur le test EIT

\begin{tabular}{l|c|c|c}
\hline & Faible & Moyen & Élevé \\
\hline Moyenne âge (écart type) & 33,23 & 38,25 & 33,1 \\
& $(8,21)$ & $(5,8)$ & $(9,35)$ \\
\hline Moyenne résidence au Québec - en mois & 1,13 & 1,17 &, 59 \\
& $(, 45)$ & $(, 83)$ & $(, 61)$ \\
\hline Moyenne études en français - en années & 1,07 & 3,30 & 6,89 \\
& $(0,98)$ & $(5,24)$ & $(3,77)$ \\
\hline Moyenne exposition quotidienne - en heures & 2,21 & 3,6 & 2,2 \\
& $(1,69)$ & $(1,66)$ & $(1,4)$ \\
\hline Moyenne usage quotidien - en heures & 3,37 & 3,12 & 2,67 \\
& $(3,33)$ & $(2,18)$ & $(2,01)$ \\
\hline
\end{tabular}

\section{L'efficacité communicative}

Pour répondre aux questions de recherche 2 et 3 portant sur le niveau d'aisance perçue et de compréhensibilité des participants, nous nous sommes appuyés sur les scores attribués par les 13 juges sur l'aisance perçue à l'oral et la compréhensibilité. Nous avons d'abord évalué la fiabilité interjuges au moyen d'alpha de Cronbach et avons observé un taux d'accord très élevé pour nos deux construits, dans les deux conditions : aisance perçue en lecture $(0,86)$, aisance perçue en production spontanée $(0,89)$, compréhensibilité en lecture $(0,89)$ et compréhensibilité en production spontanée $(0,90)$. La valeur de ces coefficients est conforme à celles rapportées dans des recherches antérieures utilisant des mesures subjectives d'aisance à l'oral et de compréhensibilité (p.ex. Derwing et Munro, 1997; Derwing et coll., 2004; Derwing et coll., 2006 et French et coll., 2020). Ayant établi une forte fiabilité interjuges, nous avons pu répondre aux questions de recherche 2 et 3 en calculant les scores moyens obtenus par nos participants pour les deux construits, en lecture à voix haute et en production spontanée.

\section{L'aisance perçue}

Notre deuxième question visait à décrire le niveau d'aisance perçue de nos participants dans deux conditions (lecture à voix haute et production spontanée). Les moyennes globales présentées au Tableau 4 indiquent que les résultats se situent au centre de l'échelle, et que par conséquent, les participants sont perçus comme n'étant ni très 
fluides (les résultats auraient alors été plus près de 1), ni très peu fluides (les moyennes globales se seraient alors rapprochées du 9). On voit également que les participants sont perçus comme ayant une plus grande aisance à l'oral lorsqu'ils font une lecture à voix haute que lorsqu'ils ont à produire à un discours spontané. L'écart entre les scores les plus bas et les plus élevés suggère que le groupe est également hétérogène en aisance perçue, et ce, dans les deux conditions.

\section{Tableau 4}

Scores sur l'aisance perçue à l'oral dans les deux conditions

\begin{tabular}{lcc}
\hline & $\mathrm{M}(E T)$ & Min.-Max. \\
\hline Lecture à voix haute & $4,9(1,4)$ & $2,8-8,5$ \\
Production spontanée & $5,8(1,4)$ & $2,9-8,6$ \\
\hline
\end{tabular}

Afin de mieux faire sens de ces résultats très hétérogènes, nous avons de nouveau séparé le groupe en trois sous-groupes en fonction de la médiane en aisance perçue dans la condition de lecture à voix haute $(4,55)$ : groupe faible $(5,04$ à 8,54$)$, groupe moyen $(4,15$ à $5,03)$ et groupe fort $(2,81$ à 4,14$)$. Nous les avons également divisés en trois en fonction de la médiane obtenue dans la tâche de production spontanée $(5,65)$ : groupe faible $(6,73$ à $8,61)$, groupe moyen $(5,23$ à 6,72$)$ et groupe élevé $(2,94$ à 5,22$)$. La Figure 3 permet de dégager un constat plus nuancé de la performance des participants. Les juges ont en réalité perçu que la majorité des participants $(\mathrm{n}=21)$ était plutôt fluide quand on leur demandait de lire à voix haute, ces participants ayant tous reçu un score moyen inférieur à 5. Par contre, 10 participants ont été perçus comme étant peu fluides dans cette même tâche (étendue des scores de ce sous-groupe : 5,8 à 8,56). Pour ce qui est de la production spontanée, ce ne sont que les participants du sous-groupe le plus fort qui ont un score inférieur à 5 , et qui sont donc perçus comme ayant une certaine aisance à l'oral dans cette condition. Les participants du sous-groupe moyen s'éloignent du score de 5 , alors que les participants du groupe le plus faible se rapprochent du 9 et sont donc perçus très peu fluides à l'oral.

\section{Figure 3}

Résultats en aisance perçue dans les deux conditions chez les sous-groupes faible, moyen et élevé

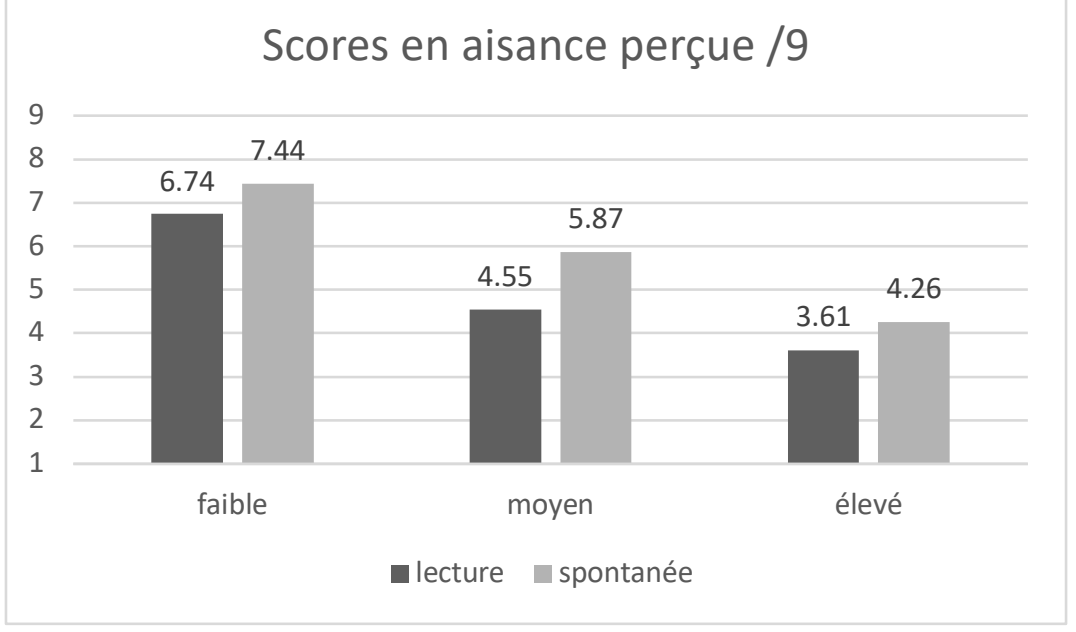


Nous nous sommes interrogés sur les caractéristiques personnelles des participants des trois sous-groupes dans les deux conditions (voir Tableau 5). On note que les caractéristiques des participants dans les deux conditions ne sont pas les mêmes. En ce qui concerne la tâche de lecture à voix haute, on constate que la seule différence marquante entre les participants des trois sous-groupes est, comme c'était le cas pour la compétence globale, le nombre d'années d'expérience antérieure d'apprentissage du français. En effet, les participants appartenant au sous-groupe qui a reçu les meilleurs jugements dans cette condition, ont étudié, en moyenne, pendant plus de 5 ans que les participants des deux autres sous-groupes. Par contre, cette même tendance n'est pas observée dans la condition spontanée. En effet, les participants qui ont obtenu des résultats faibles et élevés ont rapporté avoir étudié le français pendant plus de 4 ans avant de commencer le PILI. Les participants jugés les plus forts passent environ deux heures de plus à utiliser le français au quotidien et également deux heures de plus à être exposés au français que les participants les plus faibles, en revanche ils diffèrent peu des participants jugés comme moyen sur ces deux aspects.

Tableau 5

Caractéristiques des participants faibles, moyens et forts en aisance perçue dans deux conditions

\begin{tabular}{lcccccc}
\hline & \multicolumn{3}{c}{ Lecture à voix haute } & \multicolumn{3}{c}{ Production spontanée } \\
\cline { 2 - 7 } & Faible & Moyen & Élevé & Faible & Moyen & Élevé \\
\hline Moyenne âge (écart type) & 32,6 & 38,36 & 32,1 & 35,4 & 36,55 & 31,3 \\
& $(6,9)$ & $(7,72)$ & $(8,83)$ & $(4,43)$ & $(10,29)$ & $(8,11)$ \\
\hline Moyenne résidence au Québec - & 1,11 & 1,05 & 0,7 &, 89 & 1,14 & 0,85 \\
en mois & $(, 58)$ & $(, 47)$ & $(0,8)$ & $(, 63)$ & $(, 1)$ & $(, 82)$ \\
\hline Moyenne études en français - en & 2,05 & 1,58 & 7,13 & 4,17 & 2,09 & 4,46 \\
années & $(3,01)$ & $(2,17)$ & $(4,74)$ & $(5,44)$ & $(2,77)$ & $(4)$ \\
\hline Moyenne exposition quotidienne & 2,23 & 2,18 & 3,33 & 1,78 & 2,46 & 3,47 \\
- en heures & $(1,84)$ & $(1,58)$ & $(1,43)$ & $(1,68)$ & $(1,6)$ & $(1,37)$ \\
\hline Moyenne usage quotidien - en & 3,15 & 2,11 & 4,1 & 1,98 & 3,18 & 4,09 \\
heures & $(2,88)$ & $(1,54)$ & $(3,1)$ & $(1,39)$ & $(2,82)$ & $(3,1)$ \\
\hline
\end{tabular}

\section{La compréhensibilité}

Pour ce qui est de notre troisième question de recherche concernant le niveau de compréhensibilité de nos participants dans une tâche de lecture à voix haute et une tâche de production spontanée, le Tableau 6 indique des tendances très similaires à ce qui a été observé au Tableau 4 concernant l'aisance. Ces résultats sont peu surprenants puisque, comme expliqué précédemment, l'aisance perçue et la compréhensibilité sont deux construits fortement corrélés (voir notamment Derwing et coll., 2008). De manière générale, les moyennes globales révèlent que les participants terminant le PILI sont perçus comme ayant des habiletés ni excellentes ni déplorables en compréhensibilité. Les écarts importants entre les scores les plus bas et les scores les plus élevés montrent de nouveau que le groupe est également très hétérogène sur ce construit. 


\section{Tableau 6}

Scores en compréhensibilité dans les deux conditions

\begin{tabular}{lcc}
\hline & $\mathrm{M}(E T)$ & Min.-Max. \\
\hline Lecture à voix haute & $4,7(1,7)$ & $2,4-9$ \\
Production spontanée & $5,2(1,4)$ & $2,3-8,1$ \\
\hline
\end{tabular}

Nous avons de nouveau divisé les participants en trois groupes selon la médiane obtenue en compréhensibilité sur la tâche de lecture à voix haute $(4,44)$ : groupe faible $(5,38$ à 8,99$)$, groupe moyen $(3,51$ à 5,37$)$ et groupe fort $(2,40$ à 3,50$)$. La même manipulation a été faite à partir de la médiane obtenue sur la production spontanée $(5,05)$ : groupe faible $(5,85$ à 8,11$)$, groupe moyen $(4,59$ à 5,84$)$ et groupe élevé $(2,34$ à 4,58$)$. Ces regroupements nous permettent d'obtenir un portrait différent du groupe. On constate à la Figure 4 que dans les deux conditions, seul le groupe le plus faible a été considéré peu compréhensible, avec un score moyen s'éloignant du centre de l'échelle et se rapprochant d'un score de 7. En fait, un participant a même été jugé totalement incompréhensible en lecture recevant un score de 9. Les participants des deux autres sous-groupes sont perçus comment étant relativement compréhensibles, voire même compréhensibles pour les participants les plus forts. Dans le cas de ces deux sous-groupes, ils ont reçu des scores plus sévères lorsqu'ils devaient s'exprimer spontanément à l'oral.

\section{Figure 4}

Résultats en compréhensibilité dans les deux conditions chez les sous-groupes faible, moyen et élevé

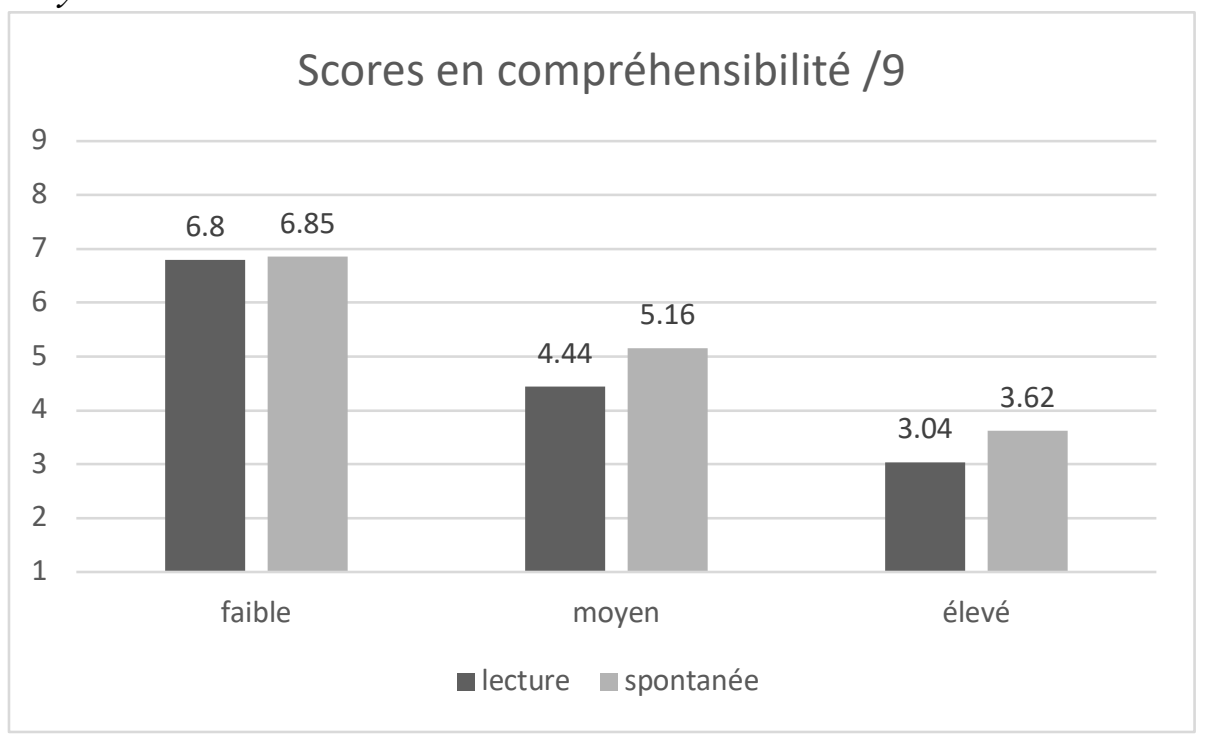

Nous nous sommes de nouveau attardés aux caractéristiques personnelles des sousgroupes de participants pour chacune des conditions. Le Tableau 7 permet de constater que les mêmes tendances s'observent dans les deux conditions. Les participants jugés comment étant les plus compréhensibles, tant dans la tâche de lecture que dans la production spontanée, ont une plus longue expérience antérieure d'apprentissage du français que les participants ayant reçu un score moyen ou faible. Par ailleurs, les participants du groupe le 
plus fort habitent au Québec depuis moins de mois que les participants de deux autres groupes.

Tableau 7

Caractéristiques des participants faibles, moyens et forts en compréhensibilité dans les deux conditions

\begin{tabular}{lcccccc}
\hline & \multicolumn{3}{c}{ Lecture à voix haute } & \multicolumn{3}{c}{ Production spontanée } \\
\cline { 2 - 7 } & Faible & Moyen & Élevé & Faible & Moyen & Élevé \\
\hline Moyenne âge (écart type) & 34,70 & 34,10 & 34,64 & 36,4 & 33,82 & 33,30 \\
& $(9,74)$ & $(6,15)$ & $(8,9)$ & $(8,81)$ & $(6,48)$ & $(9,49)$ \\
\hline Moyenne résidence au & 1,37 & 1,29 &, 51 & 1,14 & 0,95 & 0,8 \\
Québec - en mois & $(, 34)$ & $(, 86)$ & $(, 34)$ & $(, 54)$ & $(, 56)$ & $(0,8)$ \\
\hline Moyenne études en français - & 1,82 & 1,58 & 6,8 & 2,41 & 2,89 & 5,35 \\
en années & $(2,9)$ & $(1,32)$ & $(4,94)$ & $(3,53)$ & $(4,52)$ & $(4,19)$ \\
\hline Moyenne exposition & 2,33 & 2,45 & 2,88 & 3,95 & 2,6 & 3,13 \\
quotidienne - en heures & $(1,73)$ & $(1,71)$ & $(1,64)$ & $(1,78)$ & $(1,72)$ & $(1,42)$ \\
\hline Moyenne usage quotidien - & 3,23 & 3,37 & 2,71 & 2,3 & 4,06 & 2,8 \\
en heures & $(2,88)$ & $(3,01)$ & $(2,17)$ & $(2,34)$ & $(2,88)$ & $(2,49)$ \\
\hline
\end{tabular}

Nous avons présenté les données permettant de répondre à nos trois questions de recherche. Nous allons interpréter et discuter des tendances les plus importantes ayant émergé de nos données dans la prochaine section.

\section{Discussion}

Notre étude avait pour objectif de dresser un portrait des habiletés orales des personnes immigrantes qui terminent le PILI, et qui, par conséquent, devraient être sur le point d'atteindre un niveau d'autonomie langagière leur permettant de poursuivre des études ou d'occuper un emploi en français. Pour ce faire, nous avons décrit l'étendue des connaissances du code linguistique du français en évaluant la compétence globale des 31 participants et nous avons également mesuré leur efficacité communicative en demandant à des juges de faire une appréciation de leur aisance et de leur compréhensibilité à l'oral. Nous ferons un bref résumé des tendances que nous avons relevées pour chacun des construits et proposerons des pistes d'interprétation. Nous terminerons cette section par une réflexion sur des pistes pédagogiques à explorer dans ce contexte.

La performance au EIT nous a permis de constater que le groupe était extrêmement hétérogène. Rappelons que la moyenne du groupe était de 67,19/120, que le score le plus faible était de 20 et le plus élevé de 107. En mettant les descripteurs du niveau 8 en relation avec nos résultats, nos observations suggèrent que peu de participants ont en réalité pu attester qu'ils avaient développé les connaissances du code linguistique attendues à ce niveau. En effet, on s'attend, à la fin du niveau 8, à ce que l'apprenant « [e]mploie des structures de phrase variées comportant plusieurs erreurs qui toutefois n'entravent que rarement la communication », ce qui correspond à la production d'énoncés qui recevraient une note de 3 selon la grille du EIT (c.à.d., répétition précise du contenu avec quelques changements de forme, non grammaticaux ou grammaticaux, voir Figure 1). Or, la très grande majorité des participants du sous-groupe le plus faible n'a pas réussi à obtenir un 
score de trois, à partir de l'item 5 [Qu'est-ce qu'il a dit qu'il ferait aujourd'hui?], moment où il était requis de produire des énoncés complexes. Chez les participants qui ont reçu un score moyen, on constate un déclin de leur performance à partir de l'item 8 [Il est possible qu'il se mette à pleuvoir demain], partie du test où les énoncés sont plus complexes et composés de 12 syllabes. Les participants du sous-groupe le plus fort ont reçu des scores de 3 ou 4 tout au long du test, se butant, notamment, aux items de 15 syllabes formés à la négative comme l'item 18 [Elle ne commande que de la viande et ne mange aucun légume] ou à la voix passive, tel que dans l'item 22 [Le chat noir que tu as nourri a été mordu par le chien].

Ces résultats nous laissent croire que si ces finissants du PILI devaient postuler sur un emploi qui exige de faire la démonstration de sa compétence en français par la passation d'un test de langue, peu d'entre eux seraient retenus. Les participants du sous-groupe le plus fort sont ceux qui avaient une plus longue expérience d'apprentissage formel du français avant de s'inscrire au PILI. On peut raisonnablement attribuer ces résultats au type d'approche pédagogique préconisé dans les différents contextes d'apprentissage. Par exemple, les participants qui ont entamé leur apprentissage du français dans le PILI, programme qui adopte une approche communicative, centrée sur le sens et non sur la forme, ont sans doute été désavantagés par une mesure de compétence qui évalue principalement la connaissance du code linguistique. Cependant, pour les participants ayant déjà fait l'apprentissage du français en contexte de langue étrangère où on adopte encore largement une approche plutôt traditionnelle, de type Focus on FormS (voir Azimi et Joozdani, 2016), leur expérience antérieure avec la manipulation explicite du code linguistique, parfois étendue sur plusieurs années, a pu contribuer à une meilleure reconnaissance et reproduction des items retrouvés dans le EIT.

En ce qui concerne le niveau d'aisance perçue, les participants ont reçu des scores situés au centre de l'échelle, autrement dit, ils ont été perçus comme ayant un niveau d'aisance moyen. Cependant, les grands écarts entre les résultats les plus forts et les plus faibles indiquent, encore une fois, une grande hétérogénéité tant dans une tâche simple comme la lecture à voix haute que plus complexe comme une production spontanée, mais guidée. Ces résultats mis en parallèle avec le seul descripteur du niveau 8 donnant des indications quant au niveau d'aisance attendu à la fin du PILI (s'exprime en s'autocorrigeant à l'occasion), laisse entendre que quelques marques de dysfluence sont tolérées; par conséquent, un score en deçà du centre de l'échelle d'aisance perçue serait attendu en terminant le niveau 8. L'aisance à l'oral de la majorité des apprenants se situe dans cette zone dans la condition de lecture à voix haute, mais non en production spontanée. En effet, à l'instar d'études antérieures menées en anglais Lx (Javad Ahmadian et coll., 2015), les participants ont reçu des scores moyens plus élevés dans la tâche simple que dans la tâche plus linguistiquement et cognitivement complexe. Il est intéressant de noter que les participants perçus comme étant les plus fluides dans la tâche de lecture à voix haute étaient ceux qui avaient une longue expérience d'apprentissage du français. On peut émettre l'hypothèse que les connaissances antérieures explicites du français, surtout celles développées dans un contexte d'enseignement traditionnel centré sur la forme, leur ont permis de mieux émettre un message entièrement conceptualisé et formulé, en lecture. Par contre, cet avantage est disparu dans la condition de production spontanée. Ce sont plutôt les participants qui ont rapporté s'exposer et faire usage du français le plus longtemps au quotidien qui ont reçu les meilleurs scores. Cette observation nous amène 
donc à croire que les participants qui ont plus d'expérience à gérer les contraintes linguistiques et cognitives d'une interaction spontanée au quotidien se voyaient moins déstabilisés par une tâche qui créait une situation communicative dans laquelle ils devaient conceptualiser et formuler partiellement leurs énoncés.

Pour ce qui est de la compréhensibilité des finissants du PILI, les mêmes observations que celles rapportées ci-dessus peuvent être réitérées. Le groupe a reçu des scores moyens se situant au centre de l'échelle et les jugements variaient d'un extrême à l'autre, tant dans la tâche de lecture à voix haute que dans la tâche de production spontanée. Comme les participants des sous-groupes moyens et forts $(\mathrm{n}=21)$ ont obtenu, dans les deux conditions, des scores se situant entre le début et le centre de l'échelle, on peut penser que leur performance correspond raisonnablement à ce qui est attendu à ce niveau ([e]mploie des structures de phrase variées comportant plusieurs erreurs qui toutefois n'entravent que rarement la communication). Par ailleurs, tout comme les participants en anglais $\mathrm{Lx}$ de Crowther (2020), la tâche la plus simple d'un point de vue linguistique et cognitif a permis aux participants des sous-groupes moyen et fort de paraitre plus compréhensibles que dans la tâche de production spontanée, guidée qui imposait davantage de contraintes cognitives lors de la conceptualisation, la formulation et l'articulation du message. Cependant, il ne semble pas y avoir d'effet de tâches chez les participants les moins compréhensibles. Dans notre cas, les participants ayant reçu les scores les plus faibles étaient également ceux qui avaient le moins d'expérience antérieure d'apprentissage du français et donc possédaient sans doute le moins de connaissances explicites en français. Puisque la compréhensibilité est corrélée à précision grammaticale et lexicale (Trofimovich et Isaacs, 2012), on peut donc penser que pour émettre un discours qui soit perçu comme relativement compréhensible dans quelconque situation de communication, les apprenants doivent avoir atteint un seuil critique minimal de connaissances explicites du français. Cette hypothèse mériterait d'être explorée dans des études ultérieures. Il est également intéressant de constater, qu'au contraire de ce qui a été observé pour l'aisance perçue, un usage important du français au quotidien ne semble pas être associé à la performance spontanée jugée très compréhensible. En effet, c'est encore une fois, l'expérience antérieure d'apprentissage du français qui semble faire les apprenants du sous-groupe jugés le plus fort se démarquer des deux autres. Il importe donc que la recherche continue d'explorer le rôle que jouent les connaissances antérieures explicites dans la production d'un message compréhensible dans diverses tâches de production orale.

Ainsi, les observations combinées sur nos trois construits, nous laissent croire que ce n'est pas l'ensemble des 31 participants qui, au terme de leur formation linguistique, auraient atteint le seuil d'autonomie langagière requis pour intégrer une communauté de pratique qui fonctionne en français. Nos résultats laissent entrevoir que peu d'entre eux réussiraient à obtenir un emploi qui aurait comme exigence à l'embauche de pouvoir « communiquer à l'oral de façon efficace » ou de passer un test de compétence linguistique. Nos résultats corroborent aussi l'opinion de participants, qui, après avoir terminé le PILI, percevaient que leur compétence à l'oral ne leur permettait pas de prendre part avec confiance à un ensemble de situations informelles ou professionnelles du quotidien (Amireault et Lussier, 2008; Carrier-Giasson, 2017). Alors que le IRCC (2011) avait observé une corrélation positive entre la compétence globale perçue de finissants du LINC et le temps de résidence élevé au Canada, nos résultats pointent vers une autre caractéristique influençant les habiletés orales : la durée d'apprentissage du français 
antérieure à l'immigration. Comme peu d'études se sont intéressées aux adultes en contexte d'immigration, nous nous tournons vers les études qui ont exploré le rôle des connaissances antérieures sur le développement langagier en contexte de séjour linguistique à l'étranger pour interpréter le rôle de cette variable, puisque ces deux contextes, bien que différents, partagent ces caractéristiques : 1) l'apprentissage s'effectue en situation d'immersion dans la langue cible (au contraire des cours en contexte de langue étrangère) et 2) des occasions d'apprentissage sont présentes tant en salle de classe que dans le milieu social, c'est-à-dire « sur le tas ».

DeKeyser (2010) n'a observé aucun gain substantiel chez ses 16 apprenants fauxdébutants américains qui ont pris part à un séjour linguistique de 6 semaines en Argentine. DeKeyser explique ce résultat par le fait que les apprenants auraient dû minimalement posséder un certain niveau de connaissances explicites (ou connaissances déclaratives) afin de pouvoir fonctionner au quotidien dans langue cible et ainsi avoir la chance de maximiser le potentiel de procéduraliser leurs connaissances linguistiques à l'aide du contact intense avec la langue cible. Nos résultats semblent faire écho à cette observation puisque nos participants qui ont obtenu un score moyen inférieur à la moyenne au EIT (mesure de connaissances du code linguistique) ont systématiquement été perçus comme étant très peu fluides et très peu compréhensibles, tant en lecture à voix haute qu'en production spontanée. Cependant, étant donné notre petit échantillon, la relation entre le score au EIT et les scores en aisance perçue ainsi qu'en compréhensibilité, n'a pu être établie à l'aide de statistiques inférentielles. Cette relation mériterait d'être davantage étudiée dans des études ultérieures puisque les tendances que nous avons observées laissent entendre qu'il serait bénéfique d'encourager la participation à un programme linguistique et culturel avant même d'immigrer dans la société d'accueil. Pour le moment, cette possibilité est offerte par le gouvernement du Québec, en proposant le PILI en format à distance, mais il serait pertinent d'envisager la possibilité de donner des sessions intensives en présentiel dans des milieux qui recrutent un nombre important de travailleurs qualifiés.

Finalement, bien que l'on puisse s'étonner des résultats relativement faibles d'un nombre important de participants qui finissent leur formation linguistique, il importe de préciser que la performance à l'oral de nos participants les plus faibles n'est pas différente de celle d'autres apprenants de l'anglais en contexte d'immigration au Canada. À titre d'exemple, la Figure 5 présente une transcription de la performance à l'oral du participant de Duff (2012, p.5), deux ans après avoir immigré au Canada et y avoir suivi des cours d'anglais à temps plein. À l'instar de nos participants perçus comme étant les plus faibles (voir Figure 6), la production de l'apprenant dans une tâche de production relativement simple (décrire une image) est caractérisée par une majorité d'énoncés simples, parfois agrammaticaux et par de nombreuses pauses silencieuses et remplies et d'autoreformulations. 


\section{Figure 5}

Extrait de la performance à l'oral après deux ans d'instruction formelle à temps plein dans la communauté cible

The picture on (x) on the beach ( 9.0 seconds). ((laugh)). I don't know what's happen. Maybe the people they go to - on vacation. (4.0)...(8.0) (x) cameraman? ...Yeah. He's take- he:: he took a picture. I think maybe the lady (4.0) she's going drown on- in the water, and he (1.0) need(s) some help. (4.8) ... (11.0) Ah this man I thin(k) he- he's not happy I think because all the children play (0.6) to make a noisy. (3.0) Ah I dunno...

[The picture is on a beach. I don't know what's happening. Maybe the people are on vacation. There's a camera man taking a picture. I think maybe the lady is going to drown in the water and she needs some help. I think this man is not happy because all the children are playing noisily. I don't know. ...]

Figure 6

Extrait de la performance sur la tâche spontanée d'une participante qui termine le PILI

Bonjour (1.0). Réservation possible pour les groupes de vingt ou quatorze personnes (2.0) après dix (1.0) sept heure (2.0) jeudi, vendredi et samedi seulement (2.0). chaque (uhm) des menus (uhm) entrées (1.0), plates (uhm) principal, désert (3.0) vingt-neuf, vingt-neuf dollars (2.0) et quatre-dix-neuf pour personne - par personne.

\section{Retombées pédagogiques}

Un constat surprenant qui se dégage de nos résultats est la très grande hétérogénéité du groupe sur nos trois mesures d'habiletés orales. Les grands écarts peuvent sans doute s'expliquer en grande partie par les conditions de gestion du PILI : il est possible de passer au prochain niveau, et ce, même si les objectifs n'ont pas été atteints; de plus, de grandes contraintes administratives sont imposées pour faire changer un apprenant de niveau, s'il a mal été évalué lors du test de classement. Burns et Roberts (2010) remarquent que les programmes linguistiques offerts aux nouveaux arrivants sont souvent créés de manière à en faciliter la gestion par les administrateurs, reléguant au second plan la prise en compte des processus acquisitionnels et socioaffectifs qui influencent les parcours d'apprentissage. La gestion du PILI ne semble pas faire exception. Dans de telles conditions, on peut donc retrouver au niveau terminal des personnes appartenant à deux profils bien distincts: celles qui ont commencé dans un niveau inférieur et qui ont cheminé dans le PILI sans peut-être même avoir atteint les objectifs du niveau, et celles qui y ont été placées sur la base de leur résultat obtenu au test de classement. Le second profil a possiblement les compétences attendues pour réussir le niveau, mais il est incertain que ce soit le cas pour les personnes du premier profil. Il est évident que la tâche des enseignants dans un tel contexte est ardue. Il leur incombe de continuellement proposer des occasions d'apprentissage différenciées pour atteindre les objectifs associés au niveau dont ils sont responsables.

Une façon possible de différencier l'enseignement tout en favorisant le développement des habiletés orales est de proposer à l'ensemble du groupe un travail sur une même intention de communication à l'oral, mais d'imposer différentes contraintes cognitives pour la réaliser. Par exemple, pour une même tâche à l'oral, on peut différencier le temps de planification et de préparation, le nombre de répétitions possibles ou encore le 
temps donné pour accomplir la tâche en fonction des capacités des apprenants. En manipulant régulièrement ces paramètres, on crée ainsi des occasions leur permettant de graduellement apprendre à gérer les contraintes cognitives inhérentes à toutes situations de communication de la vie réelle. Plus l'apprenant progressera dans son contrôle de la charge cognitive associée à la prise de parole spontanée, meilleure sera sa préparation pour s'exprimer de manière fluide et compréhensible dans les situations du quotidien.

Une autre façon possible de gérer l'hétérogénéité dans un contexte comme celui-ci est de consacrer du temps de classe à promouvoir l'autorégulation des apprentissages, et ainsi partager avec les apprenants la responsabilité de leur développement langagier. Par exemple, comme tous les participants rapportent utiliser fréquemment le français au quotidien à l'extérieur des cours, on pourrait leur enseigner à tirer avantage de ces moments en leur indiquant comment d'autres apprenants adultes, également en situation d'immigration, ont développé leur expertise dans leur langue additionnelle. On peut ainsi enseigner aux personnes qui s'exposent au français à porter attention aux pratiques langagières de leurs interlocuteurs non seulement pour développer des connaissances culturelles, lexicales et pragmatiques (Yates et Major, 2015), mais aussi pour développer des stratégies de communication donnant l'impression que l'on a atteint une certaine aisance dans la langue (French et coll., 2017). Par ailleurs, on peut enseigner aux apprenants qui ont des interactions avec des membres de la communauté d'accueil à se servir de ces derniers comme informateurs sur la langue (Spenader, 2008) ou comme juge, par leurs réactions non verbales, de leur efficacité communicative (French et coll., 2017). Ces pratiques sont d'ailleurs celles qui sont mises en place par des apprenants qui ont développé une autonomie langagière dans la langue cible (voir Kabiri et coll., 2018).

\section{Conclusion}

Dans cette étude, nous avons dressé, pour une première fois, un portait des habiletés orales des personnes immigrantes qui terminent un programme de formation linguistique destiné à favoriser l'intégration socioprofessionnelle dans la langue de la société d'accueil. Nous avons constaté que les participants qui finissent le programme financé par le MIFI n'ont pas tous des habiletés orales égales. Alors que certains ont sans doute acquis l'autonomie langagière requise pour poursuivre des études ou travailler en français, d'autres sont bien loin d'avoir atteint ce but. Ces derniers ne sont pas différents d'autres apprenants dans la même situation (voir notamment Duff, 2012). Même si d'un point de vue acquisitionnel, il n'y a pas lieu de s'alarmer, d'un point de vue sociétal on peut tout de même se questionner à savoir si, avec des habiletés orales émergentes en français, ces derniers pourront se voir attribuer le capital linguistique nécessaire pour gagner accès à des communautés de pratiques et aux ressources symboliques et matérielles qui s'y retrouvent (Roussel, 2018).

Comme toute étude, celle-ci n'est pas sans limites. Tout d'abord, notre devis méthodologique exploratoire et descriptif ne nous permet pas de déterminer en quoi le PILI a contribué spécifiquement au développement des habiletés orales de nos participants. Par ailleurs, notre étude ne peut identifier ce qui caractérise ce groupe d'apprenants qui, au contraire de la majorité des personnes inscrites au PILI, a décidé de poursuivre jusqu'au niveau terminal. D'autres études devraient impérativement explorer ces questions pour procéder à l'amélioration continue de ce programme. Cette étude exploratoire ne permet 
pas non plus la généralisation des résultats à d'autres populations. Comme l'acquisition en contexte d'immigration est fortement influencée par la communauté d'accueil, il serait utile et important que d'autres études, reprenant nos mêmes mesures, soient menées dans d'autres milieux pour bien saisir le développement langagier des personnes immigrantes à la grandeur de la province. Par ailleurs, nos résultats sur l'efficacité communicative de nos participants sont basés sur l'évaluation de juges experts qui ont l'habitude d'interagir avec des locuteurs qui ne sont pas franco-dominants. Leurs appréciations ne reflètent peut-être pas le jugement que feraient des locuteurs qui vivent dans communautés largement homogènes du point de vue ethnoculturel. Ainsi, il serait également pertinent que des juges lambda de la communauté cible évaluent la performance à l'oral des participants au PILI, puisqu'au final ce sont eux, qui, au quotidien, jugeront l'efficacité communicative des nouveaux arrivants dans leur communauté. Finalement, notre étude a occulté un aspect important de l'efficacité communicative d'un locuteur, soit ses compétences pragmatiques à l'oral. En effet, des travaux menés auprès d'immigrants en Nouvelle-Zélande et en Australie indiquent de façon convaincante que la capacité d'un locuteur à interrompre quelqu'un, à s'excuser, à montrer son désaccord ou à taquiner de manière appropriée constitue des exemples de comportements qui contribuent au succès de l'intégration socioprofessionnelle (p.ex. Holmes, 20001 Yates, 2010, 2015). Des recherches ultérieures devraient donc impérativement décrire l'étendue de la compétence pragmatique des personnes qui terminent leur formation linguistique.

Malgré ces lacunes, notre étude a répondu à l'appel de Burns et Roberts (2010) invitant les chercheurs à documenter l'impact des programmes de formation linguistique pour nouveaux arrivants. Bien qu'ils existent dans de nombreux pays, ces programmes sont passés sous le radar de la recherche empirique. Notre étude montre qu'il faut porter davantage d'intérêt aux cadres fournis aux personnes immigrantes pour apprendre la langue de leur société d'accueil puisque le développement de leurs habiletés langagières est grandement influencé par les conditions d'apprentissage qui leur sont proposées. Dans ce contexte d'acquisition à l'âge adulte bien particulier, et encore trop peu étudié (Ortega, 2018; Plonsky, 2017), la capacité à s'exprimer efficacement dans la langue de la majorité peut faire la différence entre la marginalisation et la participation sociale et économique des personnes immigrantes.

La correspondance devrait être addressée à Suzie Beaulieu

Courriel : suzie.beaulieu@1li.ulaval.ca

\section{Notes}

${ }^{1}$ Bien que le gouvernement québécois fasse plutôt usage de l'appellation cours de francisation pour faire référence à la formation linguistique offerte aux personnes immigrantes, nous choisissons plutôt d'adopter l'appellation cours de français langue seconde (Lx), appellation attestée dans la littérature scientifique et qui a l'avantage d'être idéologiquement moins chargée (voir à ce sujet Roussel, 2018).

${ }^{2}$ Les apprenants inscrits dans un programme LINC - alphabétisation ont été exclus de l'analyse, le programme suivant un autre curriculum. 
${ }^{3}$ Notre traduction du terme commmunicative effectiveness. Comme le signale Littlemore (2003), cette expression est utilisée librement par plusieurs chercheurs, mais fait globalement référence la capacité du locuteur à se faire facilement comprendre, et par conséquent, à véhiculer avec succès ses intentions de communication. Bien que l'on puisse penser que l'efficacité communicative d'un locuteur dépende largement de sa compétence communicative (Canale et Swain, 1980; Hymes, 1972), cette relation n'a, à notre connaissance, jamais été étudiée. L'efficacité communicative d'un locuteur est plutôt largement étudiée sous l'angle de la compréhensibilité (Isaacs, 2012) ou de l'aisance à l'oral (Tavakoli, 2011).

${ }^{4}$ La présence d'un accent étranger est aussi prise en considération dans le jugement que portent les employeurs, mais ce construit est plutôt lié aux attitudes que ces derniers peuvent entretenir à l'égard du groupe ethnoculturel auquel le candidat appartient, plutôt qu'à son efficacité communicative (voir à ce sujet Derwing et Munro, 2014). La présence de l'accent étranger fera l'objet d'une étude subséquente, ancrée dans un cadre sociolinguistique.

${ }^{5}$ Toutes les productions ont été transcrites par un assistant de recherche, mais elles n'ont pas été utilisées pour répondre aux questions de recherche de la présente étude exploratoire.

\section{Références}

Adami, H. (2012). La formation linguistique des migrants adultes. Savoirs(2), 9-44.

Amireault, V., et Lussier, D. (2008). Représentations culturelles, expériences d'apprentissage du français et motivations des immigrants adultes en lien avec leur intégration à la société québécoise: étude exploratoire. Office québecois de la langue française.

Bowden, H. W. (2016). Assessing second-language oral proficiency for research: The Spanish elicited imitation task. Studies in Second Language Acquisition, 38(4), 647675.

Bradlow, A. R., Kim, M., et Blasingame, M. (2017). Language-independent talkerspecificity in first-language and second-language speech production by bilingual talkers: L1 speaking rate predicts L2 speaking rate. The Journal of the Acoustical Society of America, 141(2), 886-899.

Burns, A., et Roberts, C. (2010). Migration and adult language learning: Global flows and local transpositions. Tesol Quarterly, 44(3), 409-419.

Canale, M., et Swain, M. (1980). Theoretical bases of communicative approaches to second language teaching and testing. Applied Linguistics, 1(1), 1-47.

Carrier-Giasson, N. (2017). Le services d'enseignement du français langue seconde et leur contribution à l'intégration de personnes immigrantes allophones adultes à Saguenay. (Mémoire de maitrise). Université du Québec à Chicoutimi. https://constellation.uqac.ca/4134/1/CarrierGiasson_uqac_0862N_10300.pdf

Conseil supérieur de le langue française (2018). La francisation et l'intégration professionnelle des personnes immigrantes. http://numerique.banq.qc.ca/patrimoine/details/52327/3444424 
Crowther, D. (2020). Rating L2 speaker comprehensibility on monologic vs. interactive tasks: What is the effect of speaking task type? Journal of Second Language Pronunciation, 6(1), 96-120.

De Jong, N. H., Steinel, M. P., Florijn, A. F., Schoonen, R., et Hulstijn, J. H. (2012). Facets of speaking proficiency. Studies in Second Language Acquisition, 34(1), 5-34.

Derwing, T. M., et Munro, M. J. (2009). Putting accent in its place: Rethinking obstacles to communication. Language teaching, 42(4), 476-490.

Dustmann, C., et Van Soest, A. (2002). Language and the earnings of immigrants. ILR Review, 55(3), 473-492.

Field, A. (2015). Discovering statistics using IBM SPSS statistics (4th ed.). Sage.

French, L.M., Beaulieu, S., et Huot, D. (2017). Introduction au numéro spécial: Regard sur le développement de la compétence de communication à l'oral: récit rétrospectif d'un apprenant de français langue seconde. Revue canadienne de linguistique appliquée, 20(2), I-XXIII.

French, L. M., Gagné, N., et Collins, L. (2020). Long-term effects of intensive instruction on fluency, comprehensibility and accentedness. Journal of Second Language Pronunciation, 6(3), 380-401. http://doi.org/10.1075/jslp.20026.fre

Gauthier, C.-A. (2016). Obstacles to socioeconomic integration of highly-skilled immigrant women. Equality, Diversity and Inclusion: An International Journal, 35(1), 17-30. http://doi.org/10.1108/EDI-03-2014-0022

Gouvernement du Québec. (2011). Échelle québécoise des niveaux de compétence en français des personnes immigrantea dultes. https:/www.immigrationquebec.gouv.qc.ca/publications/fr/langue-francaise/Echelle-niveauxcompetences.pdf

Harrison, G. (2014). Accent and 'Othering' in the workplace. Dans J. M. Levis et A. Moyer (Dir.), Social dynamics in second language accent (pp. 255-272).

Hymes, D. (1972). On communicative competence. Dans J. B. Pride et J. Holmes (Dir.), Sociolinguistics: Selected Readings (Vol. 269293, pp. 269-293): Penguin.

Immigration, Réfugiés et Citoyonneté Canada (2010). Cours de langue pour les immigrants au Canada : Résultats sur la performance au CLIC.

https://www.canada.ca/fr/immigration-refugies-citoyennete/organisation/rapportsstatistiques/recherche/cours-langue-immigrants-canada-resultats-performance-clicniveau/sommaire.html

Immigration, Réfugiés et Citoyonneté Canada (2011). Évaluation du programme «Language Instruction for Newcomers to Canada» (LINC). https://www.canada.ca/fr/immigration-refugies-citoyennete/organisation/rapportsstatistiques/evaluations/programme-cours-langue-immigrants-canada2010/sommaire.html

Immigration, Réfugiés et Citoyonneté Canada (2012). Niveaux de compétence linguistique canadien : Français langue seconde pour adultes. https:/www.canada.ca/content/dam/ircc/migration/ircc/francais/pdf/pub/competenc e-linguistique.pdf

Institut de la statistique du Québec (2019). Tableau statistique canadien. http://www.stat.gouv.qc.ca/statistiques/economie/comparaisonseconomiques/interprovinciales/tableau-statistique-canadien.pdf 
Isaacs, T. (2012). Disentangling accent from comprehensibility. Bilingualism: Language and Cognition, 15(4), 905-916.

Javad Ahmadian, M., Tavakoli, M., et Vahid Dastjerdi, H. (2015). The combined effects of online planning and task structure on complexity, accuracy and fluency of L2 speech. The Language Learning Journal, 43(1), 41-56.

Laliberté, J.-W. (2019). Language skill acquisition in immigrant social networks: Evidence from Australia. Labour Economics, 57, 35-45.

Lebrun, L. A. (2012). Effects of length of stay and language proficiency on health care experiences among immigrants in Canada and the United States. Social science \& medicine, 74(7), 1062-1072.

Lindemann, S. (2003). Koreans, Chinese or Indians? Attitudes and ideologies about nonnative English speakers in the United States. Journal of sociolinguistics, 7(3), 348364.

Littlemore, J. (2003). The communicative effectiveness of different types of communication strategy. System, 31(3), 331-347.

Omar, N. H., Manaf, A. A., Mohd, R. H., Kassim, A. C., et Aziz, K. A. (2012). Graduates' employability skills based on current job demand through electronic advertisement. Asian Social Science, 8(9), 103.

Ortega, L., Iwashita, N., Norris, J., et Rabie, S. (2002). An investigation of elicited imitation tasks in crosslinguistic SLA research. Paper presented at the Second Language Research Forum, Toronto, Canada.

Ortiz, L. A., Region-Sebest, M., et MacDermott, C. (2016). Employer perceptions of oral communication competencies most valued in new hires as a factor in company success. Business and Professional Communication Quarterly, 79(3), 317-330.

Plante, J. (2011). Intégration des immigrants formés à l'étranger au marché du travail canadien: facteurs déterminants de la réussite: Statistique Canada, culture, tourisme et Centre de la statistique de l'éducation.

Minsitère de l'Immigration, de la Diversité et de l'Inclusion. (2015a). Ensemble, nous sommes le Québec: Stratégie d'action en matière d'intégration, de participation et d'inclusion.

http://www.mifi.gouv.qc.ca/publications/fr/dossiers/Strategie_ImmigrationParticipa tionInclusion.pdf

Minsitère de l'Immigration, de la Diversité et de l'Inclusion. (2015b). Stratégie de mesure de la participation des Québécoises et Québécois des minorités ethnoculturelles aux différentes sphères de la vie collective. http://www.mifi.gouv.qc.ca/publications/fr/recherchesstatistiques/Strategie_mesure_participation.pdf

Minsitère de l'Immigration, $\overline{d e}$ la Francisation et de l'Intégration (2019). Programme d'intégration linguistique pour les immigrants. http://www.mifi.gouv.qc.ca/publications/fr/divers/Pili.pdf

Ramjattan, V. A. (2019). Raciolinguistics and the aesthetic labourer. Journal of Industrial Relations, 61(5), 726-738.

Sato, T., et McNamara, T. (2019). What counts in second language oral communication ability? The perspective of linguistic laypersons. Applied Linguistics, 40(6), 894916. 
Statistique Canada (2017). Québec, V [Subdivision de recensement], Québec et Québec [Province] (tableau). Profil du recensement, Recensement de 2016. https:/www12.statcan.gc.ca/census-recensement/2016/dp$\mathrm{pd} /$ prof/details/page.cfm?Lang $=\mathrm{F} \& \mathrm{Geo} 1=\mathrm{CSD} \&$ Code $1=2423027 \& \mathrm{Geo} 2=\mathrm{CD} \& \mathrm{Cod}$ e2 $=2423 \&$ SearchText $=$ quebec $\&$ SearchType $=$ Begins $\&$ SearchPR $=01 \& B 1=A 1 \& T A$ $\mathrm{BID}=1 \&$ type $=0$

Suzuki, S., et Kormos, J. (2019). Linguistic dimensions of comprehensibility and perceived fluency: An investigation of complexity, accuracy, and fluency in second language argumentative speech. Studies in Second Language Acquisition, 1-25.

Tavakoli, P. (2011). Pausing patterns: Differences between L2 learners and native speakers. ELT Journal, 65(1), 71-79.

Tracy-Ventura, N., McManus, K., Norris, J. M., et Ortega, L. (2014). Repeat as much as you can: Elicited imitation as a measure of oral proficiency in L2 French. Dans P. Leclercq, A. Edmonds et H. Hilton (Dir.). Measuring L2 proficiency: Perspectives from SLA, pp.143-166. Multilingual Matters.

Wu, S. L., et Ortega, L. (2013). Measuring global oral proficiency in SLA research: A new elicited imitation test of L2 Chinese. Foreign Language Annals, 46(4), 680-704.

Yan, X., Maeda, Y., Lv, J., et Ginther, A. (2016). Elicited imitation as a measure of second language proficiency: A narrative review and meta-analysis. Language Testing, 33(4), 497-528. 


\section{Annexe A \\ Liste des indicateurs décrivant les attentes en production orale au niveau 8 de l'Échelle québécoise des niveaux de compétence en français des personnes immigrantes adultes}

\section{INDICATEURS}

1. Raconte un événement en utilisant adéquatement le passé composé, l'imparfait et généralement le plus-que-parfait.

- $в$ Hier soir, un triplex a pris feu à côté de chez moi. Les pompiers sont arrivés, mais, il y avait des locataires qui avaient déjà quitté l'immeuble. »

2. Emploie correctement des verbes appropriés pour exprimer des sentiments et des opinions.

= Aimer, détester, adorer, plaire, préférer, croire, penser, trouver, être certain de

3. Utilise le subjonctif pour exprimer des souhaits et des sentiments.

- E Je voudrais que vous preniez le temps."

E se suis content que tu sois là. *

4. Utilise un vocabulaire assez étendu se rapportant à des sujets dintérêt général.

5. Commence à maîtriser le vocabulaire usuel de son champ professionnel ou de son domaine d'études.

6. Emploie des structures de phrase variées comportant plusieurs erreurs qui toutefois n'entravent que rarement la communication.

" \& J'ai participé dans une activité... » (J'ai participé à une activité.)

- 8 J'espère que tu puisses dormir ce soir. .

(J'espère que tu pourras dormir ce soir.)

E s Il m'a coupé mes cheveux. * (II m'a coupé les cheveux.)

= ESi j'aurais su, j'aurais pas venu. *

(Si j'avais su, je (ne) serais pas venu.)

7. Utilise les pronoms relatifs courants pour faire des phrases complexes.

= 8 C'est la personne dont je te parlais. $\$$

8. Evite la répétition en utilisant les pronoms personnels compléments appropriés.

E Ens

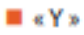

9. Résume les propos de quelqu'un.

E A la suite d'une réunion, d'un échange

10. Décrit de façon générale ses tâches professionnelles courantes.

" * Dans mon pays, j'enseignais les mathématiques au secondaire. J'avais trois classes. Il fallait faire la discipline, les préparations de cours et les corrections des examens. :

11. Emet des commentaires sur des faits d'actualité et les justifie.

12. Donne son opinion sur des sujets divers.

E * Pour ma part... *

E. A mon avis... *

* D’après moi....

* En ce qui me concerne... *

13. Donne son opinion et la justifie à l'aide d'arguments. Echange au quotidien, micro-trottoir : " * Personnellement, j'ai l'impression que.... *

* * Je (ne) suis pas d'accord avec cette prise de position parce que....

14. Négocie la solution d'un problème, propose des compromis.

" * Il vaudrait mieux que....

E Et si tu faisais... *

" * Je suis d'accord pour... mais à une condition... *

15. Tient une conversation au téléphone.

16. Formule à l'occasion des paraphrases pour vérifier ou confirmer des informations.

= * Si je comprends bien, tu dis que... *

17. S'exprime en s'autocorrigeant à l'occasion. 
Annexe B

Tâche de lecture à voix haute (adaptée de Lindeman, 2003)

Consignes : Imaginez maintenant que vous travaillez pour un bistro. Votre patron vous demande également de faire le nouvel enregistrement du message d'accueil du bistro.

Lisez le message pour vous familiariser avec son contenu ( 2 minutes). Une fois que vous serez prêt, nous commencerons l'enregistrement. Il n'y aura qu'un seul enregistrement.

Bienvenue au Bistro du Monde. Nous sommes actuellement fermés. Nous vous invitons à nous rappeler pendant nos heures d'ouverture, soit du lundi au jeudi de 10 à $21 \mathrm{~h}$ ou du vendredi au dimanche de $9 \mathrm{~h}$ à $23 \mathrm{~h}$. Vous pourrez alors réserver une table ou une salle qui conviendra à toutes les occasions. Nous vous invitons à consulter notre site internet pour plus d'informations. Merci et au plaisir de vous accueillir chez nous ! 


\section{Annexe C}

Tâche spontanée, mais guidée (adaptée de Dupere, 2018)

Consignes : Imaginez qu'un client appelle au Bistro. Il aimerait savoir s'il est possible de réserver le Bistro du Monde pour une fête de bureau.

Votre collègue a laissé des notes sur ce qu'il faut répondre dans ce contexte.

- Il faut d'abord donner toutes les informations en $A$, puis terminer en posant la question en $B$.

Partie A

- Réservation possible (groupe de 20 à 40 personnes)

- Après $17 \mathrm{~h}$ (jeudi, vendredi, samedi)

Choix de trois menus (entrée, plat principal, dessert, digestif)

Partie B $29,99 \$$ personne

- Demander la date et le nombre de personnes

Vous disposez de deux minutes pour organiser vos idées.

L'assistante de recherche sera votre partenaire pour le jeu de rôle.

Un seul enregistrement aura lieu. 